\title{
RECURRENT RANDOM WALKS WITH ARBITRARILY LARGE STEPS
}

\author{
BY L. A. SHEPP
}

Communicated by G. A. Hunt, February 10, 1964

Introduction. The random walk generated by the distribution function (d.f.), $F$, is the sequence $S_{n}=X_{1}+\cdots+X_{n}$, of sums of independent and $F$-distributed random variables. If $P\left\{\left|S_{n}\right|<1\right.$, i.o. $\}=1$, $F$ is called recurrent. ${ }^{1}$ If $F$ is not recurrent, $P\left\{\left|S_{n}\right| \rightarrow \infty\right\}=1$ [1], and $F$ is called transient. This note contains a proof that there are recurrent distributions with arbitrarily large tails. This assertion was made without proof in [2], where it is shown that for convex distributions, such behavior cannot take place.

1. Comparing random walks. We shall prove the following theorem.

Theorem. If $\epsilon=\epsilon(x)$ is defined for $x \geqq 0$, and $\epsilon(x) \rightarrow 0$, as $x \rightarrow \infty$, then there is a recurrent distribution function $F$, for which, for some $x_{0}$,

$$
1-F(x)=F(-x) \geqq \epsilon(x), \quad x \geqq x_{0} .
$$

This result may be restated in the following way. For any d.f. $G$, there is a recurrent d.f. $F$, and a sample space $W$ on which sequences $X_{n}=X_{n}(w), Y_{n}=Y_{n}(w), n=1,2, \cdots$, may be defined so that for each $w \in W$,

$$
\left|Y_{n}(w)\right|<\left|X_{n}(w)\right|, \operatorname{sign} Y_{n}(w)=\operatorname{sign} X_{n}(w), n=1,2, \cdots,
$$

where $Y_{n}, n=1,2, \cdots$, are independently $G$-distributed, and $X_{n}$, $n=1,2, \cdots$, are independently $F$-distributed. Considering $G$ transient, we have

$$
P\left\{\left|Y_{1}+\cdots+Y_{n}\right| \rightarrow \infty,\left|X_{1}+\cdots+X_{n}\right|<1 \text {, i.o. }\right\}=1
$$

We remark that $F$ cannot be chosen convex. If $F$ is (eventually) convex, and $1-F(x)=F(-x) \geqq 1-G(x)=G(-x)$, where $G$ is transient, then $F$ is also transient [2].

The idea of the proof of the theorem is to move out the mass of $G$ and bunch it up, leaving large gaps, so that the remaining steps somehow cancel themselves out.

2. Proof of the cancellation theorem. For symmetric $F$, the condition that $F$ be recurrent is a tail condition [2], and may be stated

\footnotetext{
${ }^{1}$ i.o. or infinitely often here means for infinitely many $n=1,2, \cdots$.
} 
in terms of the characteristic function, $\phi(z)=\int \cos x z d F(x)$, as

$$
\int_{0}^{1}(1-\phi(t))^{-1} d t=\infty \text {. }
$$

Since any function $\epsilon$ of our theorem is majorized by a piecewise constant function, continuous except at integers, and decreasing to zero, we may restrict ourselves to functions of this type.

We shall prove the stronger assertion.

Lemma. If $p_{n}>0, n=1,2, \cdots, \sum p_{n}<\infty$, and $0<y_{n} \uparrow \infty$, are given, then

$$
\int_{0}^{1}\left(\sum p_{n}\left(1-\cos x_{n} t\right)\right)^{-1} d t=\infty,
$$

for some $x_{n} \geqq y_{n}, n=1,2, \cdots \cdot$

Assuming the lemma, choose $x_{0}$ so that $\epsilon\left(x_{0}^{-}\right) \leqq 1 / 2$, and set $p_{n}=\epsilon\left(y_{n}^{-}\right)-\epsilon\left(y_{n}^{+}\right)$, where $y_{n}, n=1,2, \cdots$, are the jumps of $\epsilon$ to the right of $x_{0}$. We define $F$ to have mass $p_{n}$ at $\pm x_{n}, n=1,2, \cdots$, provided by the lemma. The remaining mass of $F, 1-2 \epsilon\left(x_{0}^{-}\right)$ $=1-2 \sum p_{n}$ is placed at zero. As defined, $F$ is symmetric, and

$$
1-F(x)=\sum_{x_{n} \geqq x} p_{n} \geqq \sum_{y_{n} \geqq x} p_{n} \geqq \epsilon(x),
$$

for $x>x_{0}$. By (2.2), we have (2.1), and $F$ is recurrent.

To prove the lemma, assume that $n_{0}=0<n_{1}<\cdots<n_{k}$ have already been defined (start at $k=0$ ), and that $x_{1}, \cdots, x_{n_{k}}$ have been chosen so that $x_{n} \geqq y_{n}, n=1,2, \cdots, n_{k}$, and

$$
\int_{0}^{1}\left(\sum_{n \leqq n_{k}} p_{n}\left(1-\cos x_{n} t\right)+2 \sum_{n>n_{k}} p_{n}\right)^{-1} d t \geqq k .
$$

We shall show that it is possible to choose $n_{k+1}>n_{k}$ and $x_{n_{k}+1}, \cdots$, $x_{n_{k+1}}$, so that $x_{n} \geqq y_{n}, n_{k}<n \leqq n_{k+1}$, and so that (2.3) holds with $k$ replaced by $k+1$. Having shown this, $x_{n}$ are then inductively defined for all $n=1,2, \cdots$ and $x_{n} \geqq y_{n}$. Moreover, for any $k$,

$$
\begin{aligned}
\int_{0}^{1}\left(\sum p_{n}\left(1-\cos x_{n} t\right)\right)^{-1} d t & \\
& \geqq \int_{0}^{1}\left(\sum_{n \geqq n_{k}} p_{n}\left(1-\cos x_{n} t\right)+2 \sum_{n>n_{k}} p_{n}\right)^{-1} d t,
\end{aligned}
$$

and by (2.3), (2.2) follows. 
We now show that $n_{k+1}=m$, and $x_{n_{k}+1}=x_{n_{k}+2}=\cdots=x_{n_{k+1}}=x$ can be defined, where $x \geqq y_{n_{k+1}}$, and $m>n_{k}$. This is a consequence of the following assertion, where $a=n_{k}$ is fixed

$$
\begin{aligned}
\lim _{m \rightarrow \infty} \lim _{x \rightarrow \infty} \int_{0}^{1}( & \sum_{n \unlhd a} p_{n}\left(1-\cos x_{n} t\right) \\
& \left.+\left(\sum_{n=a+1}^{m} p_{n}\right)(1-\cos x t)+2 \sum_{n>m} p_{n}\right)^{-1} d t=\infty .
\end{aligned}
$$

Since $\sum_{n \leqq a} p_{n}\left(1-\cos x_{n} t\right) \leqq c t^{2}$ for some fixed $c>0$, we find that (2.5) is a consequence of (2.6),

$$
\lim _{\epsilon \rightarrow 0} \lim _{x \rightarrow \infty} \int_{0}^{2 \pi}\left(t^{2}+1-\cos x t+\epsilon^{2}\right)^{-1} d t=\infty .
$$

Writing $\int_{0}^{2 \pi}=\sum_{n=1}^{x} \int_{2 \pi(n-1) \leqq t x<2 \pi n}$, and using the fact that $1-\cos$ $r \leqq c r^{2}$, for some $c>0$, we have only to show that

$$
\lim _{\epsilon \rightarrow 0} \lim _{x \rightarrow \infty} x^{-1} \sum_{n=1}^{x} \int_{0}^{2 \pi}\left(n^{2} x^{-2}+r^{2}+\epsilon^{2}\right)^{-1} d r=\infty .
$$

Noting that $a_{1}^{2}+a_{2}^{2}+a_{3}^{2} \leqq\left(a_{1}+a_{2}+a_{3}\right)^{2}$ for $a_{i} \geqq 0, i=1,2,3$, and integrating, the sum in (2.7) is at least

$$
\begin{aligned}
& x^{-1} \sum_{n=1}^{x} \int_{0}^{1}\left(n x^{-1}+r+\epsilon\right)^{-2} d r \\
& \quad=\sum_{n=1}^{x} x^{-1}\left(n x^{-1}+\epsilon\right)^{-1}\left(n x^{-1}+1+\epsilon\right)^{-1} .
\end{aligned}
$$

For $\epsilon<1$, this sum is at least $\sum_{n=1}^{x}(n+\epsilon x)^{-1} 3^{-1}$. Now, as $x \rightarrow \infty$,

$$
\sum_{n=1}^{x}(n+\epsilon x)^{-1}=\log x(1+\epsilon)-\log \epsilon x+O(1) .
$$

Hence the first limit in (2.7) is $\log 1+\epsilon^{-1}$, which, indeed, tends to infinity with $\epsilon^{-1}$. This proves the assertions.

\section{REFERENCES}

1. K. L. Chung and W. H. J. Fuchs, On the distribution of sums of random variables, Mem. Amer. Math. Soc. No. 6 (1951), 12 pp.

2. L. A. Shepp, Symmetric random walk, Trans. Amer. Math. Soc. 104 (1962), 144-153.

Bell Telephone laboratories, Murray Hill, New Jersey 